\title{
sh-HNF1A-AS1 reduces the epithelial-mesenchymal transition and stemness of esophageal cancer cells
}

\author{
Zheng WANG, Yan-Feng HUANG*, Ling YU, Yue JIAO \\ Department of Thoracic Surgery, Cancer Hospital of China Medical University, Liaoning Cancer Hospital and Institute, Shenyang, China \\ ${ }^{*}$ Correspondence: huangyanfengdoc@aliyun.com
}

Received November 2, 2021 / Accepted January 17, 2022

\begin{abstract}
HNF1A-AS1 is a cancer-related lncRNA. Elevated expression of HNF1A-AS1 has been observed in various malignancies including esophageal squamous cell carcinoma (ESCC). However, the role of HNF1A-AS1 in ESCC has not been fully understood. This study aimed to investigate the potential role of HNF1A-AS1 in ESCC. Expression of HNF1A-AS1, miRNA (miR)-298, and transcription factor 4 (TCF4) was detected using qRT-PCR. The interactions between HNF1A-AS1 and miR-298 or miR-298 and TCF4 were evaluated. Short hairpin RNAs (shRNAs) were used to knock down HNF1A-AS1 (sh-HNF1A-AS1). The EMT and stemness of ESCC cells were detected. HNF1A-AS1 was overexpressed in ESCC tumor tissues and cells. miR-298 was validated as a direct target of HNF1A-AS1. sh-HNF1A-AS1 significantly inhibited EMT and stemness of ESCC cells. The high expression of miR-298 significantly inhibited the expression of TCF4, and further inhibited the expression of N-cadherin and stemness-related genes. Animal experiments showed that sh-HNF1A-AS1 significantly inhibited tumor growth and increased the level of miR-298 in tissues. In conclusion, knockdown of HNF1A-AS1 could inhibit EMT and stemness by regulating the miR-298/TCF4 axis.
\end{abstract}

Key words: HNF1A-AS1; miR-298; esophageal squamous cell carcinoma; epithelial-mesenchymal transition; stemness; transcription factor 4

Esophageal squamous cell carcinoma (ESCC) is a highly aggressive malignant disease, which is the major pathological type of esophageal cancer in Chinese patients [1]. The median overall survival (OS) of ESCC patients is 4-6 months [2]. High aggressiveness and early metastasis contribute to the poor prognosis of ESCC.

Increasing evidence has demonstrated that lncRNAs had a regulating role in cancer development and progression, functioning as both tumor suppressors and oncogenes in a variety of cancer types [3]. HNF1A-AS1 is a cancer-related IncRNA. Elevated expression of HNF1A-AS1 has been observed in various malignancies including ESCC [1, 4, 5]. Zhao et al. indicated that upregulation of HNF1A-AS1 contributed to cell proliferation and metastasis in osteosarcoma [6]. Guo et al. found that HNF1A-AS1 was upregulated in colorectal cancer (CRC) tissues with a role in regulating CRC cell migration, invasion, and glycolysis [4]. Furthermore, the inducing role of HNF1A-AS1 on epithelial-mesenchymal transition (EMT) has been observed by Wu et al. [7]. And knockdown of HNF1A-AS1 has been demonstrated to suppress tumor cell proliferation and metastasis, thereby suppressing tumor progression $[1,8]$. But the role of HNF1AAS1 in ESCC has not been fully understood.
LncRNAs have been confirmed to function as competing endogenous RNAs (ceRNAs) to participate in modulating the expression of the downstream targets of microRNAs (miRNAs) [9]. Previous studies have revealed that HNF1AAS1 could regulate the expression of several miRNAs, including miR-214 [1], miR-17-5p [8], and miR-124 [4]. miR-298 is a molecular hub that involves in the invasiveness and metastasis of cancer cells $[10,11]$. In a study conducted by Mo et al., a lncRNA named LINC01287 could regulate the EMT phenotype in hepatocellular carcinoma (HCC) cells via miR-298/STAT3 axis [12]. The result indicated that miR-298 might involve in the process of EMT. However, whether HNF1A-AS1 could function on miR-298 to regulate EMT has not been determined before.

Therefore, the aim of this study was to explore the potential role of HNF1A-AS1 in ESCC and to detect the underlying molecular mechanism.

\section{Patients and methods}

Patients and specimens. Fifty pairs of ESCC tumor tissues and matched adjacent non-tumorous tissues were collected from patients who had undergone surgery at the 
Department of Thoracic Surgery, Cancer Hospital of China Medical University (Shenyang, China) between Sept 2016 and Dec 2017. The survival time of these 50 patients was also recorded and analyzed. The study was approved by the Ethics Committee of Cancer Hospital of China Medical University (Shenyang, China). All patients provided written informed consent.

Cell culture. In the present study, the human ESCC cell lines (EC109, EC9706, KYSE-30, and KYSE-70) and normal human esophageal epithelial cell line (HEEC) were purchased from the Type Culture Collection of the Chinese Academy of Sciences (Shanghai, China). EC109, EC9706, and HEEC were grown in DMEM (Gibco, MA, USA). KYSE-30 and KYSE-70 were cultured with RPMI-1640 (Gibco). All kinds of cells were cultured in a humidified atmosphere of $5 \% \mathrm{CO}_{2}$ at $37^{\circ} \mathrm{C}$.

Quantitative real-time polymerase chain reaction (qRT-PCR). Total RNAs were extracted from cell lines and tissues using TRIZOL reagent (Invitrogen, Carlsbad, CA, USA). RNA was reverse-transcribed using the cDNA Synthesis Kit (TaKaRa Bio, USA). qRT-PCR was performed with an ABI StepOnePlus ${ }^{\text {tw }}$ real-time PCR System (Applied Biosystems, USA). The relative gene expression was calculated using the $2^{-\triangle \Delta C t}$ method. Primers used are as follows: HNF1A-AS1， 5'-TCAAGAAATGGTGGCTAT-3' (forward primer) and 5'-GCTCTGAGACTGGCTGAA-3' (reverse primer); miR-298, 5'-GATTAGCACCGACTCTA-3' (forward primer) and 5'-GAAGCCATCTGACATAG-3' (reverse primer); TCF4, 5'-AGAAACGAATCAAAACAGCTCCT-3' (forward primer) and 5'-CGGGATTTGTCTCGGAAACTT-3' (reverse primer); GADPH, 5'-AGAAGGCTGGGGCTCATTTG-3' (forward primer) and 5'-AGGGGCCATCCACAGTCTTC-3' (reverse primer); U6, 5'-GCGCGTCGTGAAGCGTTC-3' (forward primer) and 5'-GTGCAGGGTCCGAGGT-3' (reverse primer). All experiments were performed independently in triplicate.

Transfection assay. miR-298 negative control (NC), miR-298 mimic, miR-298 inhibitor, and NC mimic were obtained from the GenePharma Company (Shanghai, China). Short hairpin RNAs (shRNAs) for HNF1A-AS1 knockdown (sh-HNF1A-AS1-1, sh-HNF1A-AS1-2, and sh-HNF1A-AS1-3) and scrambled negative control (sh-NC) were synthesized by Invitrogen (Carlsbad, CA, USA). Recombinant adenoviral vectors containing TCF4 (Ad-TCF4) and blank vectors (Ad-vectors) were constructed by the GenePharma Company.

EC109 and KYSE-70 cells were seeded in 24-well plates. The aforementioned plasmids were transfected into EC109 or KYSE-70 cells using Lipofectamine 2000 (Invitrogen) according to the manufacturer's instructions.

Dual-luciferase reporter assay and RNA binding protein immunoprecipitation (RIP). The wild type (WT) and mutant (MUT) of HNF1A-AS1 were constructed and inserted into the luciferase reporter assay vector pmirGLO (Promega Corp., Madison, WI, USA) as described previously [13]. The
Luc-TCF4-WT and Luc-TCF4-MUT were constructed using the same method. The EC109 and KYSE-70 cells were seeded overnight and co-transfected with NC mimics or miR-298 mimics. Then, a Dual-Luciferase Reporter Assay System (Promega) was used to detect the luciferase activity of cells. All assays were performed independently in triplicate.

RIP assay was performed using RNA-Binding Protein Immunoprecipitation Kit (Millipore, USA). AntiAgo2 (Millipore) was used to enrich HNF1A-AS1 and miR-298. The negative control was normal mouse Anti-IgG (Millipore). All assays were performed independently in triplicate.

Spheroid formation assay. $24 \mathrm{~h}$ after transfection, EC109 or KYSE-70 cells were collected and resuspended in $20 \mu \mathrm{l}$ of culture media containing Proliferation Supplements (Stem Cell Technologies, Canada) as described previously [14]. After 2 weeks of incubation at $37^{\circ} \mathrm{C}$, the spheres were counted and images were obtained using a light stereomicroscope (Olympus Corporation). The diameters of spheroids were measured using ImageJ Software. All assays were performed independently in triplicate.

Flow cytometric analysis of the aldehyde dehydrogenase activity. Aldehyde dehydrogenase 1 (ALDH1) activity in this study was assessed using the ALDEFLUOR kit (Stem Cell Technologies, Durham, NC, USA). After transfection, cells were suspended in $1 \mathrm{ml}$ ALDEFLUOR buffer and incubated at $37^{\circ} \mathrm{C}$ for $45 \mathrm{~min}$. And then cells were centrifuged and resuspended in $0.5 \mathrm{ml}$ ALDEFLOUR assay buffer. The $\mathrm{ALDH}^{+}$population was measured by flow cytometry (Becton Dickinson). All experiments were performed independently in triplicate.

Western blotting. The proteins extracted from tissues and cells were separated by SDS-PAGE. And then the proteins were transferred onto polyvinylidene fluoride (PVDF) membranes (Millipore, MA, USA). After being blocked for $1 \mathrm{~h}$, blots were incubated with primary antibodies overnight at $4{ }^{\circ} \mathrm{C}$. Antibodies against Snail (1:1000, Cat. \# 3895, Cell Signaling Technology, Danvers, MA, USA), E-cadherin (E-cad, 1:1000, Cat. \# 14472, Cell Signaling Technology), N-cadherin (N-cad, 1:1000, Cat. \# 14215, Cell Signaling Technology), Vimentin (Vim, 1:1000, Cat. \# 3390, Cell Signaling Technology), sexdetermining region Y-box 2 (SOX2, 1:400, Cat. \# ab79351, Abcam, Cambridge, UK), Nanog homeobox (NANOG, 1:400, Cat. \# ab80892, Abcam), octamer-binding protein 4 (OCT4, 1:400, Cat. \# ab19857, Abcam), TCF4 (1:1000, Cat. \# 2953, Cell Signaling Technology), and $\beta$-actin (1:30000, Cat. \# bsm-33036M, Bioss, Beijing, China) were incubated. Then, membranes were incubated with anti-rabbit or antimouse IgG-HRP-conjugated secondary antibodies (1:10000, Wuhan Boster Biological Technology, Wuhan, China) for 1 hour at room temperature. All experiments were performed independently in triplicate.

Tumor growth assay. Animal experiments were performed under a project license granted by the Animal Care and Use Committee of China Medical University. Fourweek-old male $\mathrm{Nu} / \mathrm{nu}$ athymic nude mice (Weitonglihua 
Biotechnology, Beijing, China) were raised under specific pathogen-free conditions. EC109 or KYSE-70 cells were transfected with sh-HNF1A-AS1 for $24 \mathrm{~h}$. Then, a total of $1 \times 10^{6}$ cells (EC109 and KYSE-70 cells with stable expression of sh-HNF1A-AS1 or control cells) were injected into the left forelimb in nude mice. Ten mice were assigned per group.

Mice in each group were sacrificed four weeks after injection, and the tumor nodules were harvested. Then, the tumor weight of each mouse was immediately measured. And the tumor tissues were collected for further analysis including qRT-PCR, Western blotting, and immunohistochemistry.

Immunohistochemistry. Formalin-fixed paraffinembedded sections $(5 \mu \mathrm{m})$ from tissues were incubated with rabbit anti-mouse primary antibodies directed against E-cad and Vim (Cell Signaling Technology) overnight at $4{ }^{\circ} \mathrm{C}$. Biotinylated goat anti-rabbit anti-immunoglobulin $\mathrm{G}$ (Wuhan Boster Biological Technology) was used as the secondary antibody. Proteins were visualized under a light microscope. Each sample was analyzed in triplicate.

Bioinformatics analysis. The potential pairing bases between HNF1A-AS1 and miR-298 were predicted by MIRDB (http://mirdb.org/). Target genes of miR-298 were predicted by TargetScan 5.1 (http://www.targetscan.org/).
Statistical analysis. The statistical analyses were conducted by SPSS 22.0 software (Chicago, USA). All results were presented as mean \pm standard deviation (SD). A Student's t-test or one-way analysis of variance (ANOVA) was used to evaluate the differences between groups. The Kaplan-Meier method was used to estimate the survival time of 50 patients with ESCC, with log-rank tests being used to assess significance. A p-value $<0.05$ was considered to be significant.

\section{Results}

The expression of HNF1A-AS1 was elevated in ESCC. The expressions of HNF1A-AS1 and miR-298 in 50 ESCC tumor tissues and matched adjacent non-tumorous tissues were detected by qRT-PCR. As shown in Figure 1, HNF1AAS1 levels were strongly increased in ESCC tumor tissues, as compared to the normal tissues ( $\mathrm{p}<0.01$, Figure $1 \mathrm{~A})$. But the miR-298 levels showed a reverse trend. The relative expression of miRNA-298 in ESCC tumor tissues was much lower than that in normal tissues $(\mathrm{p}<0.01$, Figure $1 \mathrm{~B})$. The correlation analysis indicated that the expression of HNF1A-AS1 in tumor tissues was negatively correlated with the expression of miR-298 $\left(\mathrm{R}^{2}=-0.696, \mathrm{p}<0.001\right.$, Figure $\left.1 \mathrm{C}\right)$. In addition,
A

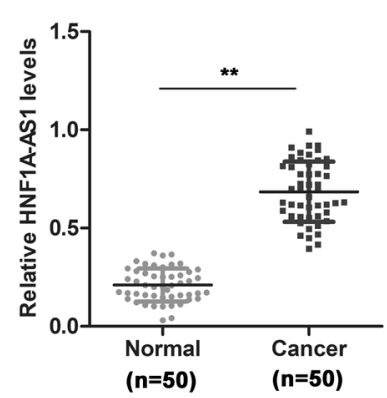

D

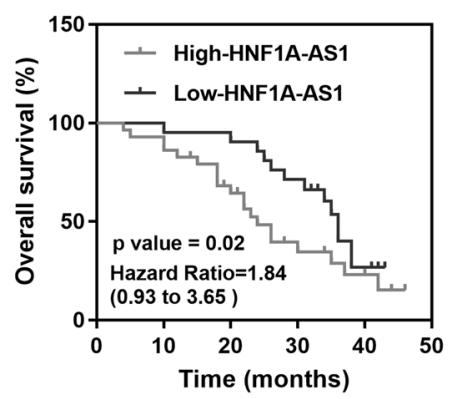

B

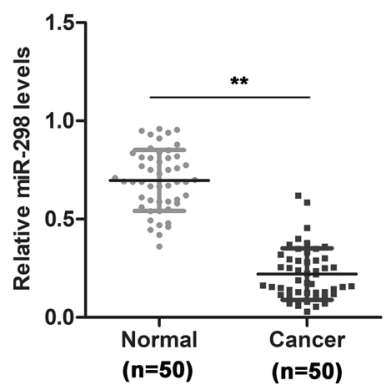

E

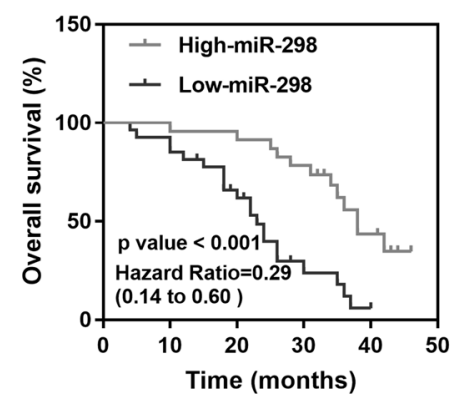

C

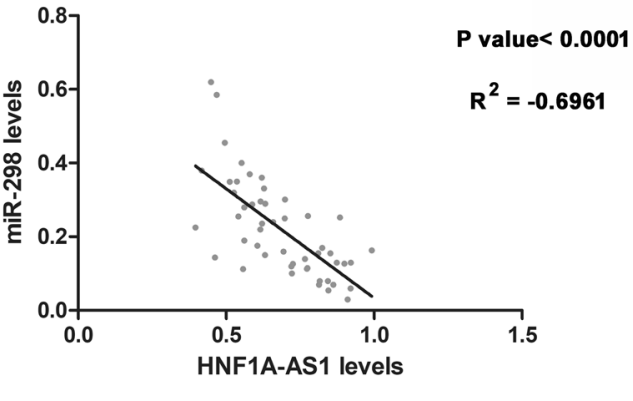

$\mathbf{F}$
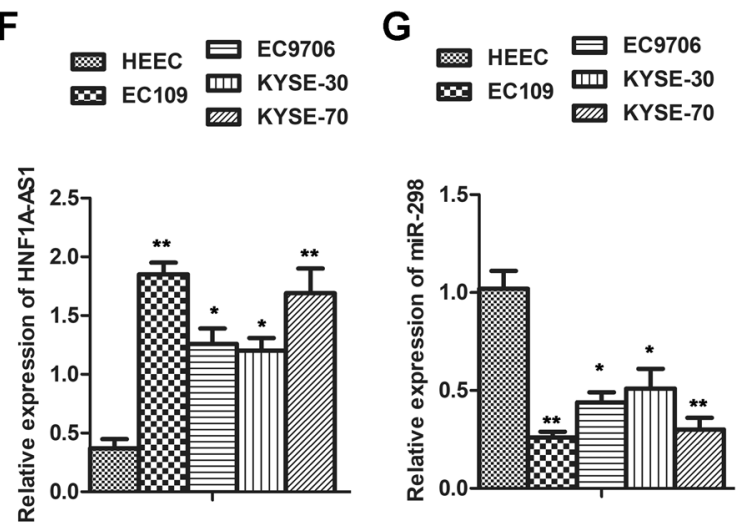

Figure 1. HNF1A-AS1 was highly expressed in esophageal squamous cell carcinoma (ESCC). A) Relative expression of HNF1A-AS1 in ESCC tumor tissues and normal tissues was detected by qRT-PCR. ${ }^{* *}$ p $<0.01$ vs. normal tissues. B) Relative expression of miR-298 in ESCC tumor tissues and normal tissues was detected by $\mathrm{qRT}$-PCR. ${ }^{* *} \mathrm{p}<0.01$ vs. normal tissues. C) The correlation analysis of HNF1A-AS1 expression and miR-298 expression in tumor tissues. D) The survival analysis of patients with a high HNF1A-AS1 expression and a low HNF1A-AS1 expression. E) The survival analysis of patients with a high miR-298 expression and a low miR-298 expression. F) Relative expression of HNF1A-AS1 in ESCC cell lines was detected by qRT-PCR. ${ }^{\star} \mathrm{p}<0.05$ vs. HEEC; ${ }^{\star *} \mathrm{p}<0.01$ vs. HEEC G) Relative expression of miR-298 in ESCC cell lines was detected by $q R T-P C R .{ }^{\star} p<0.05$ vs. HEEC; ${ }^{\star *} p<0.01$ vs. HEEC. 
patients with stage III/IV were prone to have high expression of HNF1A-AS1 and low expression of miRNA-298 (Table 1). The survival analysis of the 50 patients showed that patients with high HNF1A-AS1 expression had a shorter median survival time than those with low HNF1A-AS1 expression (24 months vs. 36 months, $\mathrm{HR}=1.84, \mathrm{p}=0.020$, Figure 1D), while patients with high miR-298 expression had a longer median survival time than those with low miR-298 expression (38 months vs. 23 months, $\mathrm{HR}=0.29, \mathrm{p}<0.001$, Figure $1 \mathrm{E}$ ).

Then, the expressions of HNF1A-AS1 and miR-298 in human ESCC cell lines (EC109, EC9706, KYSE-30, and KYSE-70) and HEEC were also detected. As expected, compared with HEEC cells, the expression of HNF1A-AS1 was much higher $(\mathrm{p}<0.05$, Figure $1 \mathrm{~F})$, while the expression of miRNA-298 was much lower in ESCC cell lines $(\mathrm{p}<0.05$, Figure 1G).

miR-298 was a direct target of HNF1A-AS1. Bioinformatics tools were utilized to predict the potential pairing bases between HNF1A-AS1 and miR-298. MIRDB showed a putative targeting site of miR-298 in the HNF1A-AS1 transcript (Figure 2A). Then, miR-298 mimic, miR-298 inhibitor, NC inhibitor, and NC mimic were transfected into EC109 or KYSE-70 cells, and the miR-298 levels were detected by qRT-PCR. As expected, the expression of miR-298 was decreased in the miR-298 inhibitor group as compared to the control group $(\mathrm{p}<0.01)$. And the expression of miR-298 was strongly increased in the miR-298 mimic group $(p<0.001$, Figures 2B, 2C). HNF1A-AS1 knockdown significantly inhibited the HNF1A-AS1 expression (Figures 2D-2F). Then, HNF1A-AS1-wt and HNF1A-AS1-mut were cloned into a dual-luciferase system. The results revealed that EC109 or KYSE-70 cells co-transfected with HNF1A-AS1-Wt and miR-298 mimics strongly decreased the luciferase activity,

Table 1. The features of $\mathbf{5 0}$ patients with esophageal squamous cell carcinoma.

\begin{tabular}{|c|c|c|c|c|c|c|c|}
\hline \multirow{2}{*}{ Features } & \multirow{2}{*}{ Cases } & \multicolumn{2}{|c|}{ HNF1A-AS1 } & \multirow{2}{*}{ p-value } & \multicolumn{2}{|c|}{ miR-298 } & \multirow{2}{*}{ p-value } \\
\hline & & High & Low & & High & Low & \\
\hline \multicolumn{2}{|l|}{ Age (year) } & & & 0.738 & & & 0.670 \\
\hline$\geq 50$ & 32 & 18 & 14 & & 14 & 18 & \\
\hline$<50$ & 18 & 11 & 7 & & 9 & 9 & \\
\hline \multicolumn{2}{|l|}{ Gender } & & & 0.536 & & & 0.266 \\
\hline Male & 24 & 15 & 9 & & 13 & 11 & \\
\hline Female & 26 & 14 & 12 & & 10 & 16 & \\
\hline \multicolumn{2}{|c|}{ Tumor size (mm) } & & & 0.598 & & & 0.982 \\
\hline$\leq 3$ & 26 & 16 & 10 & & 12 & 14 & \\
\hline$>3$ & 24 & 13 & 11 & & 11 & 13 & \\
\hline \multicolumn{2}{|c|}{ Lymphatic invasion } & & & 0.239 & & & 0.247 \\
\hline Yes & 24 & 16 & 8 & & 9 & 15 & \\
\hline No & 26 & 13 & 13 & & 14 & 12 & \\
\hline TNM stage & & & & 0.015 & & & 0.005 \\
\hline I/II & 24 & 8 & 13 & & 16 & 8 & \\
\hline III/IV & 26 & 21 & 8 & & 7 & 19 & \\
\hline
\end{tabular}

while those co-transfected with HNF1A-AS1-Mut and miR-298 mimics did not affect the luciferase activity $(\mathrm{p}<0.05$, Figures 2H, 2I). RIP assay revealed that both HNF1A-AS1 and miR-298 were in the same RNA-induced silencing complex (RISC), as these two RNAs were enriched in Ago2 immunoprecipitates compared to IgG-pellet $(\mathrm{p}<0.001$, Figures $2 \mathrm{~F}$, 2G). Next, sh-HNF1A-AS1 and/or miR-298 inhibitor were transfected into EC109 or KYSE-70 cells. After cells were transfected with sh-HNF1A-AS1, miR-298 expression was significantly increased as compared to control $(\mathrm{p}<0.01$, Figures 2J, 2K). Interestingly, sh-HNF1A-AS1 could reverse the inhibition effect of the miR-298 inhibitor on miR-298 expression. Compared with the miR-298 inhibitor group, the sh-HNF1A-AS1+miR-298 inhibitor group had a much higher miR-298 expression ( $\mathrm{p}<0.01$, Figures 2J, 2K). Collectively, those findings indicated that miR-298 could directly target HNF1A-AS1 specifically.

sh-HNF1A-AS1 inhibited EMT in ESCC cells. sh-HNF1A-AS1 and/or miR-298 inhibitor were transfected into EC109 or KYSE-70 cells to show the role of HNF1AAS1 on EMT. $48 \mathrm{~h}$ after transfecting, cell morphology was observed (Figure 3A). Cells in the control group exhibited a typical polygonal cobblestone epithelial morphology. Some cells in the miR-298 inhibitor group exhibited elongated spindle shape and cell-cell inter-contact became loose. But sh-HNF1A-AS1 could reverse this change caused by the miR-298 inhibitor in EC109 and KYSE-70 cell lines.

Western blot analysis of the EMT associated proteins showed that the epithelial biomarker E-cad was increased, while the mesenchymal biomarkers $\mathrm{N}$-cad and Vim, and Snail, an EMT driver transcription factor, were appreciably decreased in cells transfected with sh-HNF1A-AS1 $(\mathrm{p}<0.05$ vs. control, Figures 3B, 3C). But the expression of these EMT-associated proteins in cells transfected with miR-298 inhibitor had an opposite tendency $(\mathrm{p}<0.05$ vs. control, Figures 3B, 3C). As compared to the miR-298 inhibitor group, the expression of E-cad was increased and the expression of N-cad, Vim as well as Snail was '05, Figures 3B, 3C). Our results indicated that sh-HNF1A-AS1 could reverse the function of miR-298 inhibitor on EMT in both EC109 and KYSE-70 cells.

sh-HNF1A-AS1 inhibited stemness in ECSS cells. sh-HNF1A-AS1 and/or miR-298 inhibitor were transfected into EC109 or KYSE-70 cells to show the function of HNF1A-AS1 on stemness. The representative images of the spheroid formation assay are shown in Figure 4A. The diameter of a sphere in the sh-HNF1A-AS1 group was much smaller than that in the control group $(\mathrm{p}<0.05$, Figure $4 \mathrm{~A})$. Furthermore, miR-298 inhibitor strongly increased the sphere diameter ( $p<0.001$ vs. control group), which could be reversed by sh-HNF1A-AS1. The diameter of a sphere in the sh-HNF1A-AS1+miR-298 inhibitor group was much smaller than that in the miR-298 inhibitor group $(p<0.001$, Figure $4 \mathrm{~A})$. The number of $\mathrm{ALDH}^{+}$cells in each group had a similar tendency in both cell lines (Figure 4B). SOX2, NANOG, and 
A

HNF1A-AS1-MUT

5 ' -TAGgGaGgCtgagTtACGAGAATC-3'

HNF1A-AS1-WT

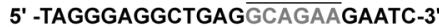

||||||

miR-298

3'-UCGUCUUCGUCCCUCCAAGAGGGU-5'

Control
NC inhibitor
$\square$ miR-298 inhibitor
NC mimic
miR-298 mimic

Control
NC inhibitor
miR-298 inhibitor
NC mimic
miR-298 mimic
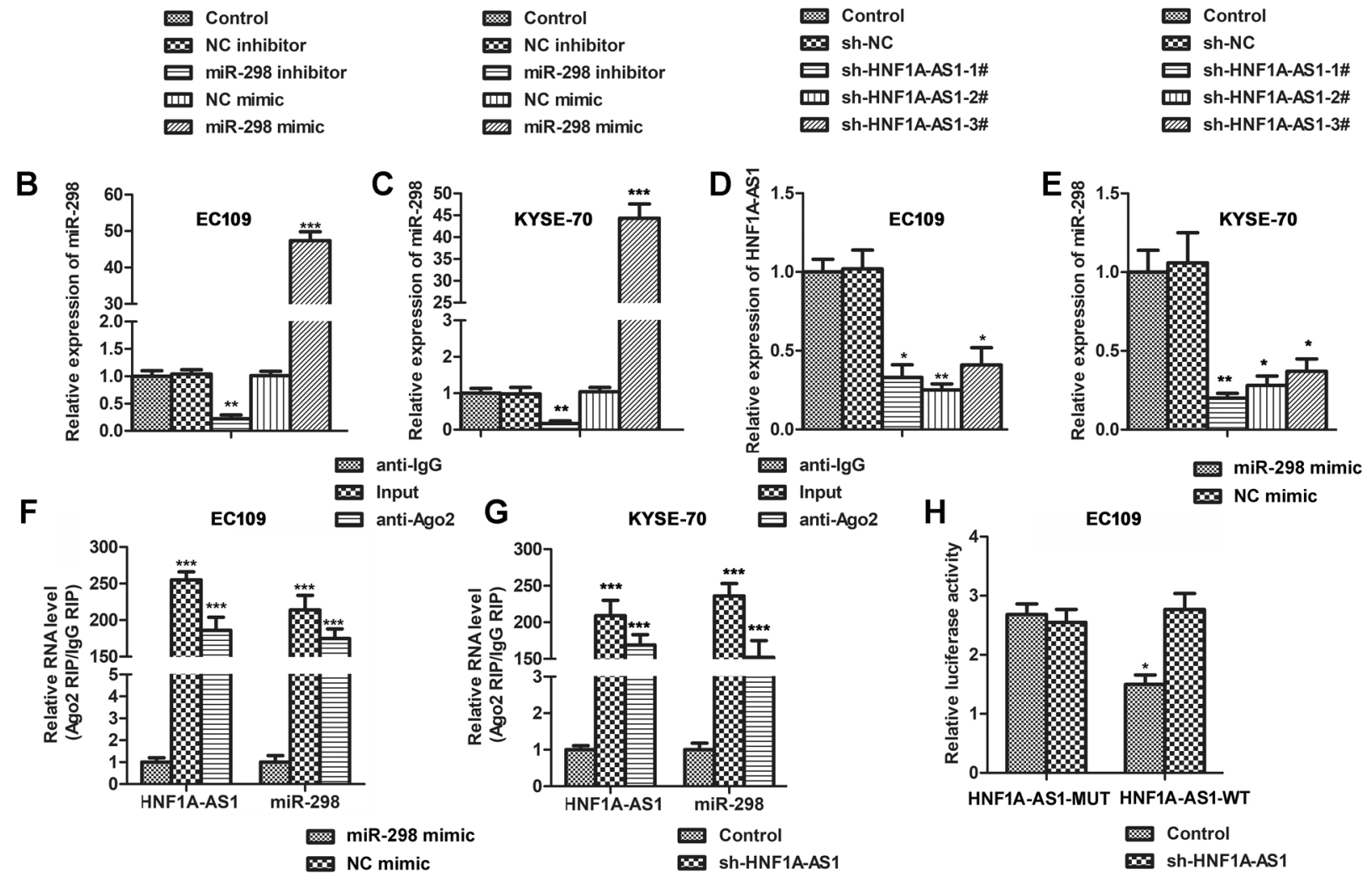

H

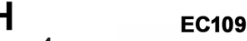

I
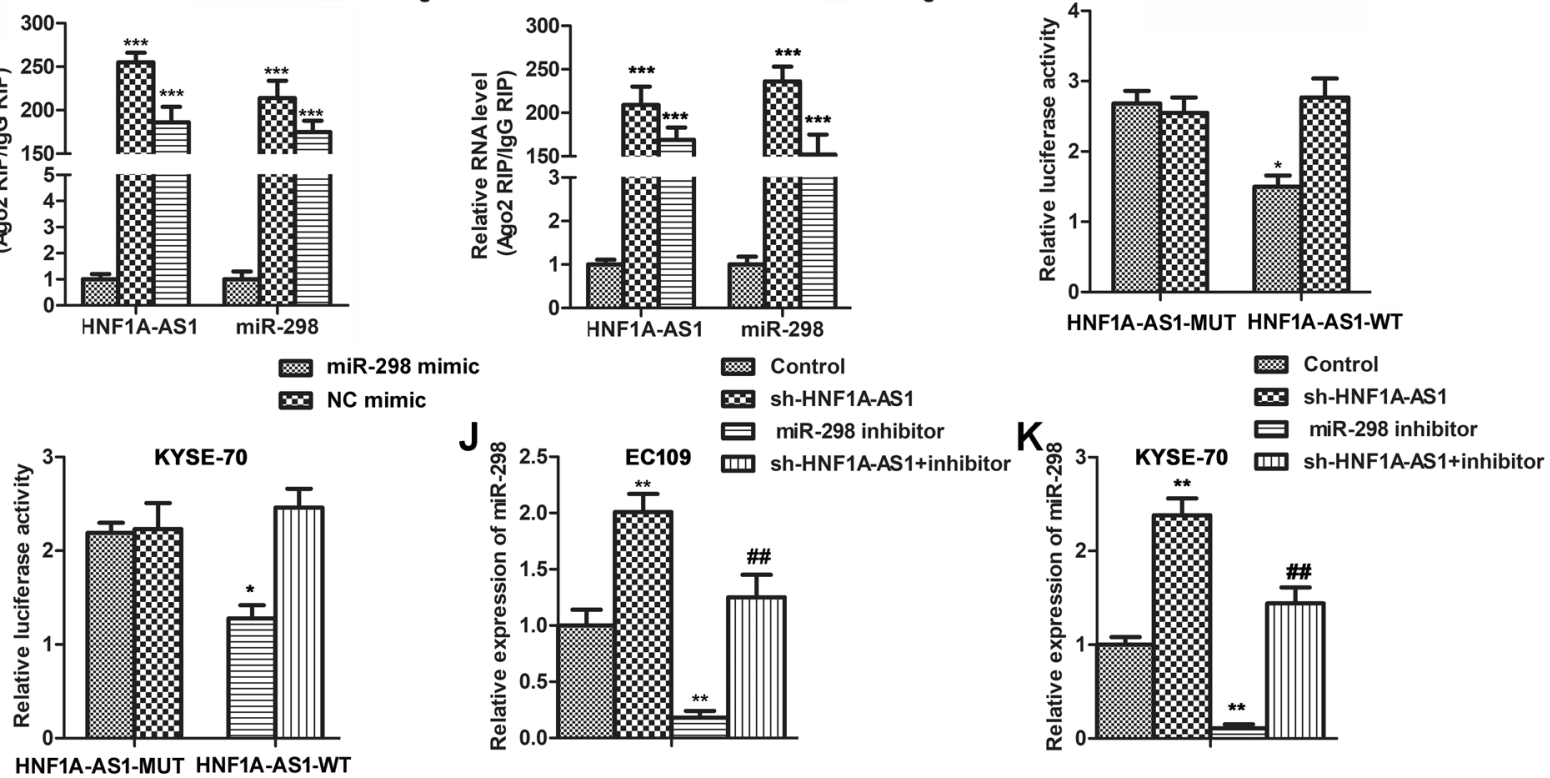

Figure 2. miR-298 was a direct target of HNF1A-AS1. A) Bioinformatics analysis showed a putative targeting site of miR-298 in the HNF1A-AS1 transcript. B) miR-298 mimic, miR-298 inhibitor, NC inhibitor, and NC mimic were transfected into EC109 cells and the miR-298 levels were detected by $q R T-P C R .{ }^{* *} p<0.01$ vs. control group; ${ }^{* *} p<0.001$ vs. control group. C) miR-298 mimic, miR-298 inhibitor, NC inhibitor, and NC mimic were transfected into KYSE-70 cells and the miR-298 levels were detected by qRT-PCR. ${ }^{* *} \mathrm{p}<0.01$ vs. control group; ${ }^{* * *} \mathrm{p}<0.001$ vs. control group. D) Three sh-HNF1A-AS1s were transfected into EC109 cells and the expression of HNF1A-AS1 was detected by qRT-PCR. ${ }^{*} \mathbf{p}<0.05$ vs. control group; ${ }^{\star *} \mathbf{p}<0.01$ vs. control group. E) Three sh-HNF1A-AS1s were transfected into KYSE-70 cells and the expression of HNF1A-AS1 was detected by qRT-PCR. ${ }^{*}$ p $<0.05$ vs. control group; ${ }^{* *} \mathbf{p}<0.01$ vs. control group. F) The RIP assays of EC109 cells found that HNF1A-AS1 and miR-298 were in the same RNA-induced silencing complex (RISC). ${ }^{* *}{ }^{*}<<0.001$ vs. anti-IgG. G) The RIP assays of KYSE-70 cells found that HNF1A-AS1 and miR-298 were in the same RNAinduced silencing complex (RISC). ${ }^{* *} \mathbf{p}<0.001$ vs. anti-IgG. H) The luciferase reporter assay was conducted to detect the luciferase activity in EC109 cells. ${ }^{\star} \mathbf{p}<\mathbf{0 . 0 5}$ vs. NC mimic. I) The luciferase reporter assay was conducted to detect the luciferase activity in KYSE-70 cells. ${ }^{*} \mathbf{p}<0.05$ vs. NC mimic. J) sh-HNF1A-AS1 and/or miR-298 inhibitor were transfected into EC109 cells. And the expression of miR-298 was detected by qRT-PCR. ${ }^{\star *}$ p $<0.01$ vs. control group; ${ }^{* *} \mathrm{p}<0.01$ vs. miR-298 inhibitor group. K) h-HNF1 A-AS1 and/or miR-298 inhibitor were transfected into KYSE-70 cells. And the expression of miR-298 was detected by qRT-PCR. ${ }^{\star *} \mathrm{p}<0.01$ vs. control group; ${ }^{* *} \mathrm{p}<0.01$ vs. miR-298 inhibitor group.

OCT4 are markers of cancer stem cells. In the present study, the levels of these proteins were detected by western blot. As shown in Figures 4C and 4D, the miR-298 inhibitor group had the highest levels of these three proteins in both cell lines ( $\mathrm{p}<0.05$ vs. control group). And sh-HNF1A-AS1 could decrease their expression. The relative levels of these proteins 
in the sh-HNF1A-AS+miR-298 inhibitor group were much lower than those in the miR-298 inhibitor group $(\mathrm{p}<0.05)$. Thus, these results demonstrated that sh-HNF1A-AS1 could suppress the stemness of EC109 and KYSE-70 cells. And this effect might relate to its role on miR-298.

TCF4 was a potential target of miR-298. Target Scan was employed to search for the potential target genes of miR-298. TCF4 was a candidate target gene (Figure 5A). The expressions of TCF4 in human ESCC cell lines (EC109,
EC9706, KYSE-30, and KYSE-70) and HEEC were detected by qRT-PCR. As expected, relative expression of TCF4 was strongly increased in ESCC cell lines ( $p<0.05$ vs. HEEC, Figure 5B). The Dual-Luciferase reporter assay revealed that EC109 or KYSE-70 cells co-transfected with TCF4-Wt and miR-298 mimics decreased luciferase activity remarkably $(p<0.01$, Figures 5C, 5D), further confirming that TCF4 was a potential target gene for miR-298. Next, sh-HNF1A-AS1 and/or miR-298 inhibitor were transfected into EC109 or
A
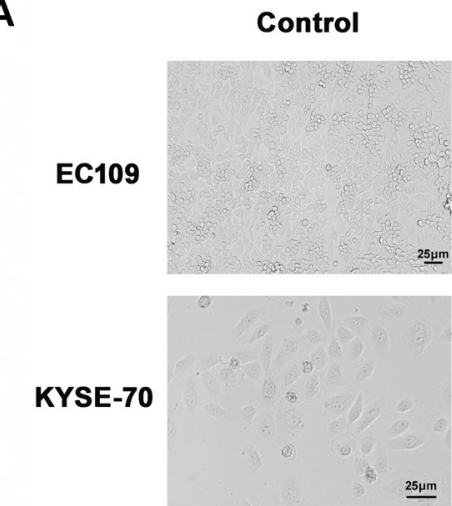

B

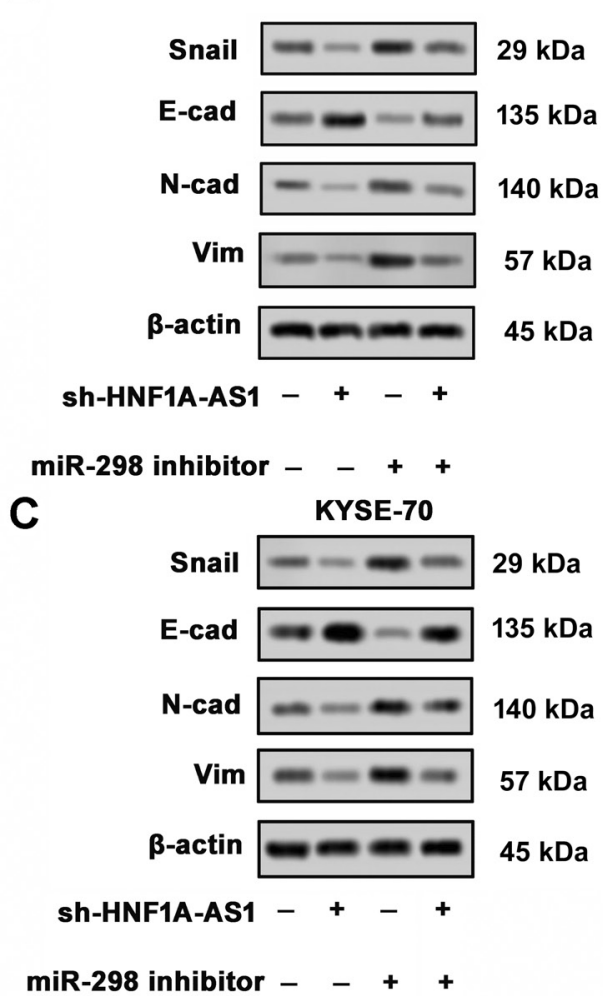

sh-HNF1A-AS1
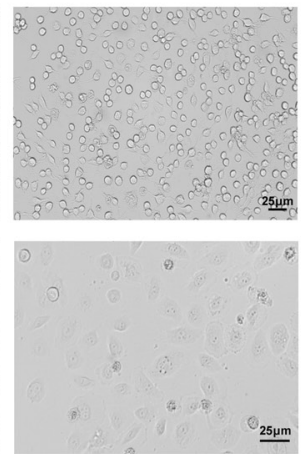

$25 \mathrm{~mm}$
miR-298 inhibitor sh-HNF1A-AS1+inhibitor
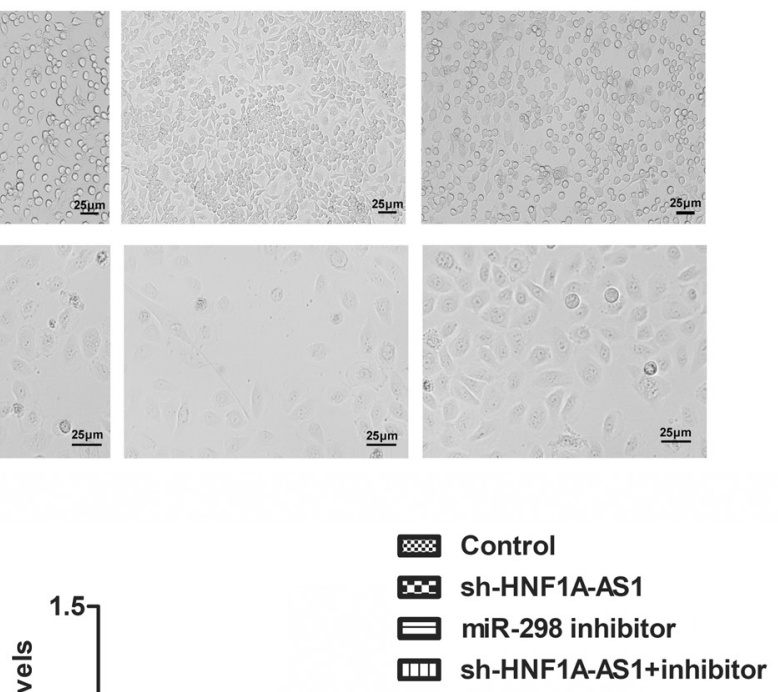

m sh-HNF1A-AS1+inhibitor
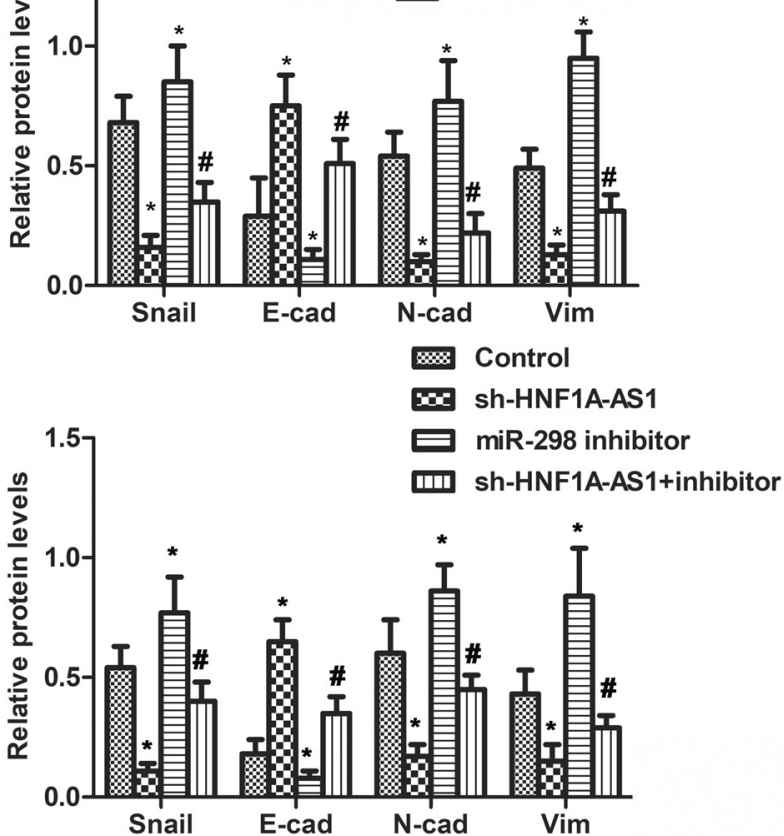

Figure 3. sh-HNF1A-AS1 inhibited epithelial-mesenchymal transition (EMT). A) sh-HNF1A-AS1 and/or miR-298 inhibitor were transfected into EC109 and KYSE-70 cells. $48 \mathrm{~h}$ after transfecting, cell morphology was observed under an invert phase-contrast microscope (200×). B) sh-HNF1 A-AS1 and/or miR-298 inhibitor were transfected into EC109 cells. The expression of EMT-associated proteins was evaluated by western blot analysis. ${ }^{*} \mathbf{p}<0.05$ vs. control group; ${ }^{*} \mathbf{p}<0.05$ vs. miR-298 inhibitor group. C) sh-HNF1 A-AS1 and/or miR-298 inhibitor were transfected into KYSE-70 cells. The expression of EMT-associated proteins was evaluated by western blot analysis. ${ }^{*} \mathbf{p}<0.05$ vs. control group; ${ }^{*} \mathbf{p}<0.05$ vs. miR-298 inhibitor group. 
KYSE-70 cells. After cells were transfected with sh-HNF1AAS1, the expressions of TCF and $\beta$-catenin were significantly decreased as compared to control ( $\mathrm{p}<0.05$, Figures $5 \mathrm{E}, 5 \mathrm{~F}$ ). However, the miR-298 inhibitor could strongly increase the TCF4 and $\beta$-catenin expressions ( $\mathrm{p}<0.05$ vs. control, Figures 5E, 5F). Furthermore, compared with the miR-298 inhibitor group, the sh-HNF1A-AS1+miR-298 inhibitor group had a much lower TCF4 expression as well as $\beta$-catenin expression $(p<0.05$, Figures $5 E, 5 F)$. These results indicated that TCF4 was a potential target of miR-298. And HNF1AAS1 could regulate the miR-298/TCF4 axis in ESCC cells.

sh-HNF1A-AS1 inhibited tumor growth in vivo. To investigate the effects of HNF1A-AS1 on tumor growth in vivo, EC109 or KYSE-70 cells were pre-treated with sh-HNF1A-AS1 and the xenograft mouse model was created. Compared to the control group, the average tumor weight in the sh-HNF1A-AS1 group was much smaller $(\mathrm{p}<0.05$, Figures 6A-6D). Consistent with the results obtained from in vitro studies, sh-HNF1A-AS1 could also decrease the expression of HNF1A-AS1 and TCF 4 ( $<<0.01$, Figures $6 \mathrm{E}-6 \mathrm{~F})$, and increase the expression of miR-298 $(\mathrm{p}<0.01$, Figures $6 \mathrm{E}, 6 \mathrm{~F})$ in vivo. Besides, the expression of stemness-related proteins (SOX2, NANOG, and OCT4) was strongly suppressed by sh-HNF1A-AS1 $(p<0.05$, Figures $6 \mathrm{G}, 6 \mathrm{H})$. And the results of immunohistochemistry revealed that sh-HNF1A-AS1 could increase the expression of $\mathrm{E}$-cad $(\mathrm{p}<0.01)$ and decrease the

A

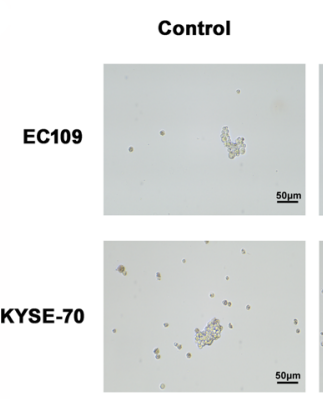

sh-HNF1A-AS1
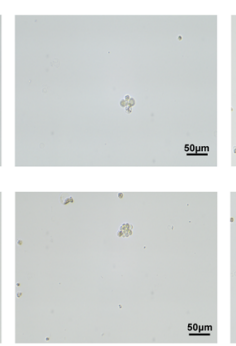

B
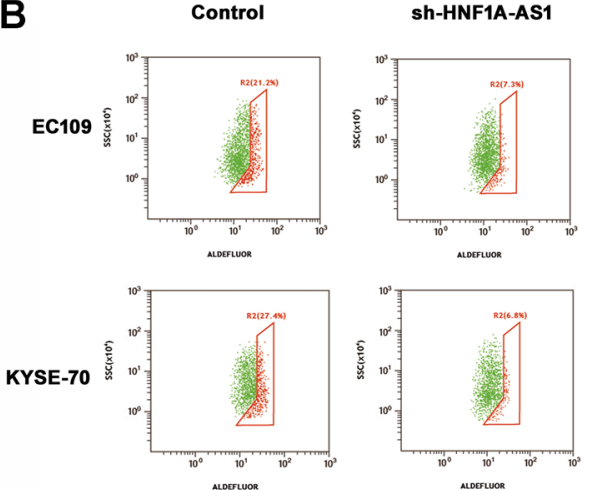

Control

sh-HNF1A-AS1

miR-298 inhibitor

m sh-HNF1A-AS1+inhibitor
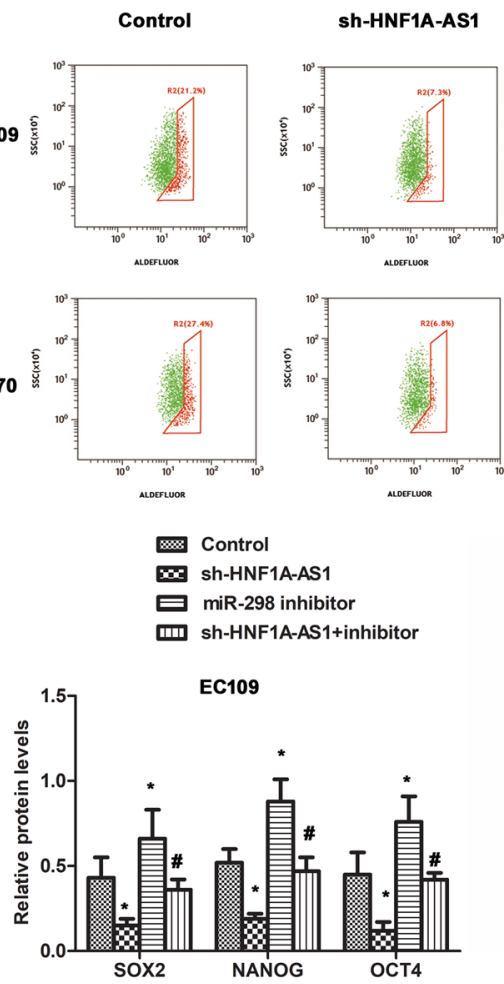

miR-298 inhibitor sh-HNF1A-AS1+inhibitor

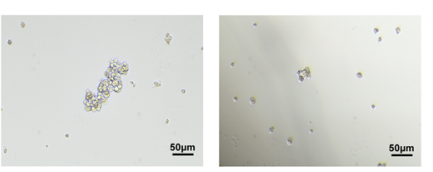

oum
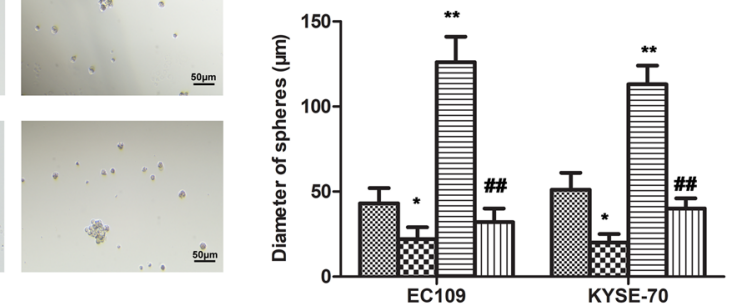

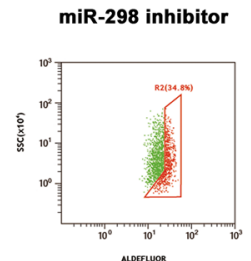

sh-HNF1A-AS1+inhibitor

C

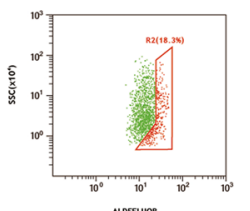

EC109
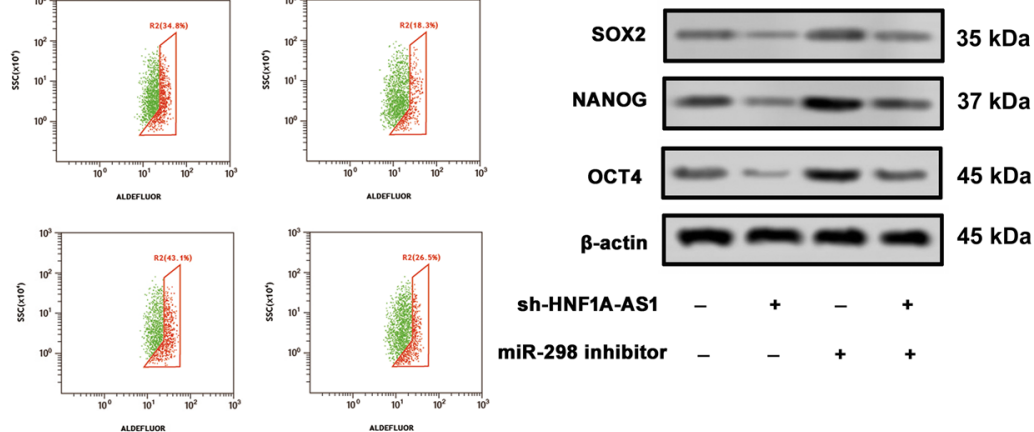

D

KYSE-70
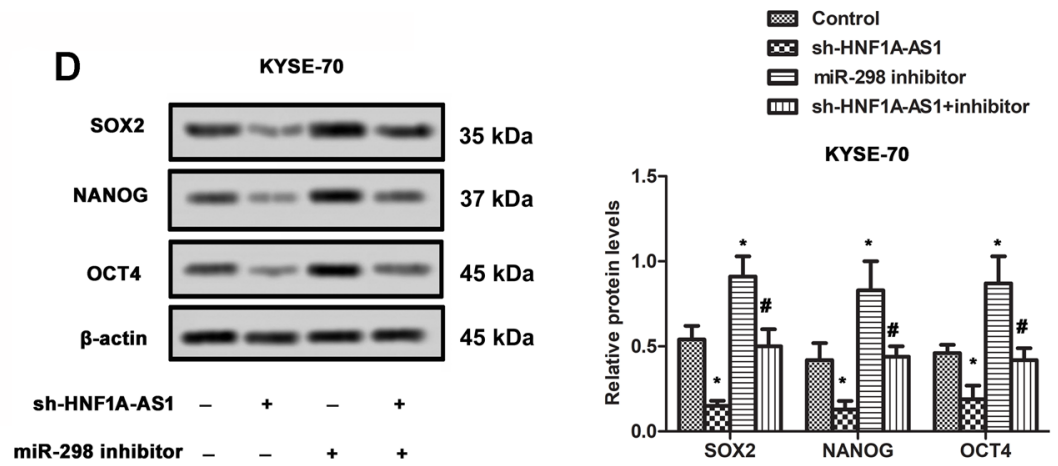

Figure 4. sh-HNF1A-AS1 inhibited stemness. A) The diameter of the sphere in each group was measured. B) The number of ALDH1 ${ }^{+}$cells in each group was detected by flow cytometric analysis. C) The expression of stemness markers in EC109 cells was detected by western blot. D) The expression of stemness markers in KYSE-70 cells was detected by western blot. ${ }^{\star} \mathrm{p}<0.05$ vs. control group; ${ }^{\star * *} \mathrm{p}<0.001$ vs. control group; ${ }^{\sharp} \mathrm{p}<0.05$ vs. miR-298 inhibitor

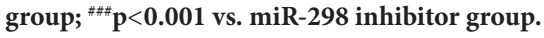




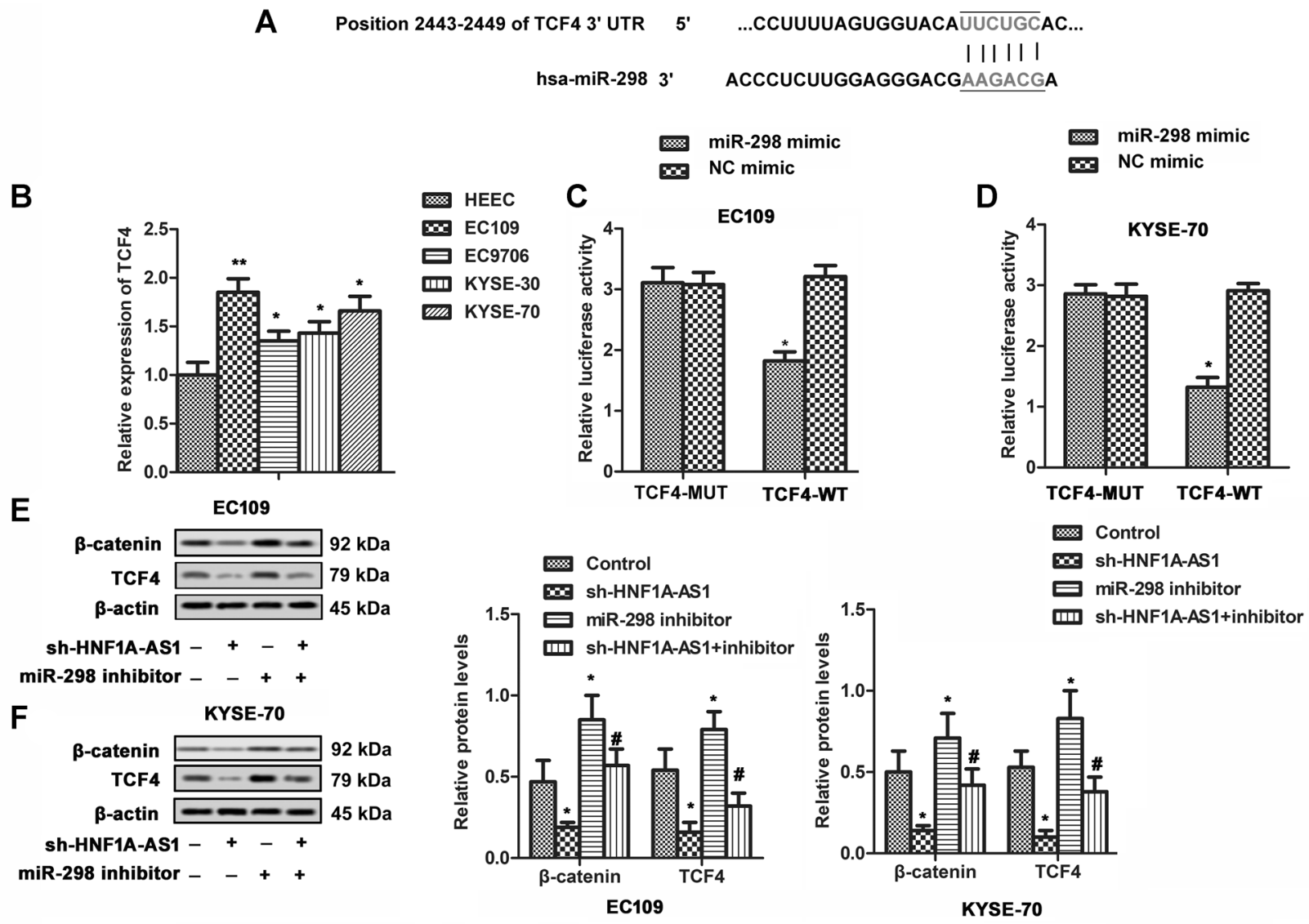

Figure 5. miR-298 affected the expression of TCF4. A) Bioinformatics analysis identified TCF4 as a candidate target gene for miR-298. B) Relative expression of TCF4 in ESCC cell lines was detected by qRT-PCR. ${ }^{\star} p<0.05$ vs. HEEC; ${ }^{* *} p<0.01$ vs. HEEC C) The luciferase reporter assay was conducted to detect the luciferase activity in EC109 cells. ${ }^{*} \mathrm{p}<0.05 \mathrm{vs}$. NC mimic. D) The luciferase reporter assay was conducted to detect the luciferase activity in EC109 cells. ${ }^{*} \mathbf{p}<0.05$ vs. NC mimic. E) sh-HNF1A-AS1 and/or miR-298 inhibitor were transfected into EC109 cells. The expressions of TCF4 and $\beta$-catenin were evaluated by western blot analysis. ${ }^{*} p<0.05$ vs. control group; ${ }^{*} p<0.05$ vs. miR-298 inhibitor group. F) sh-HNF1A-AS1 and/or miR-298 inhibitor were transfected into KYSE-70 cells. The expressions of TCF4 and $\beta$-catenin were evaluated by western blot analysis. ${ }^{\star} \mathrm{p}<0.05$ vs. control group; ${ }^{*} \mathbf{p}<0.05$ vs. miR-298 inhibitor group.

Vim level ( $\mathrm{p}<0.01$, Figures 6I, 6J) in tumor tissues. The above results showed that sh-HNF1A-AS1 played a role in EMT and stemness in ESCC, thus inhibiting tumor growth in vivo.

\section{Discussion}

HNF1A-AS1 is a newly discovered lncRNA, playing an important regulatory role in the progression of many types of human cancers including lung cancer [7], gastric cancer [5], and colorectal cancer [4]. In 2017, Wang et al. found that HNF1A-AS1 was overexpressed in ESCC [1]. And knockdown of HNF1A-AS1 could target miR-214 to inhibit cell viability and motility [1]. Consistently, we showed that HNF1A-AS1 was markedly upregulated in ESCC tumor tissues and cell lines (EC109, EC9706, KYSE-30, and KYSE-70) in the present study. The elevated expression of HNF1A-AS1 suggested that HNF1A-AS1 was involved in the pathogenesis of ESCC. Additionally, we also found that knockdown of HNF1A-AS1 could inhibit EMT and stemness by targeting miR-298/TCF4, suggesting HNF1A-AS1 is a promising therapeutic target for ESCC treatment.

In the current study, the data indicated that miR-298 expression in human ESCC cell lines was significantly downregulated compared with that in normal controls. Downregulation of miR-298 has been reported to promote cancer cell proliferation and metastasis $[10,11]$. Thus, miR-298 was regarded as a suppressor in tumor progression. LncRNAs are located upstream of miRNA, which may act as endogenous molecular sponges to compete for miRNAs, regulating miRNA expression negatively [15]. miRNAs, such as miR-124, miR-214, and miR-30b-30p, have been reported to act as direct targets of HNF1A-AS1 $[1,4,16]$. And overexpression of these miRNAs could suppress the role of HNF1AAS1 in tumor cell viability, migration, and invasion [4, 17]. In this study, miR-298 was also validated as a direct target of HNF1A-AS1 based on the following results: first, bioinformatics tools showed a putative targeting site of miR-298 in the HNF1A-AS1 transcript; second, the direct binding 

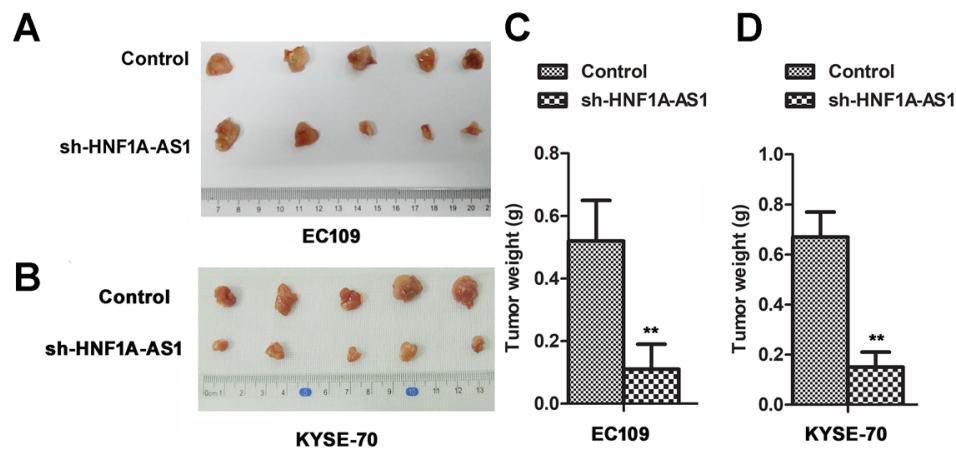

E

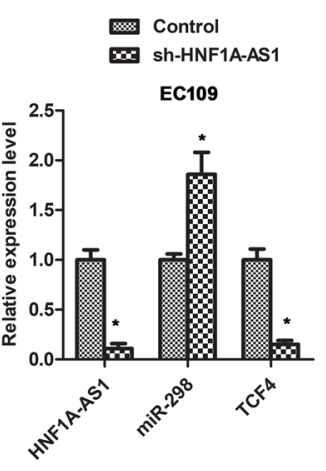

$\mathbf{F}$

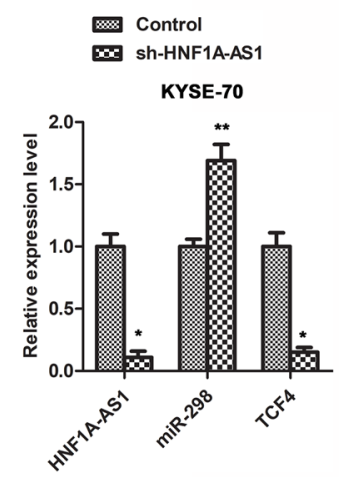

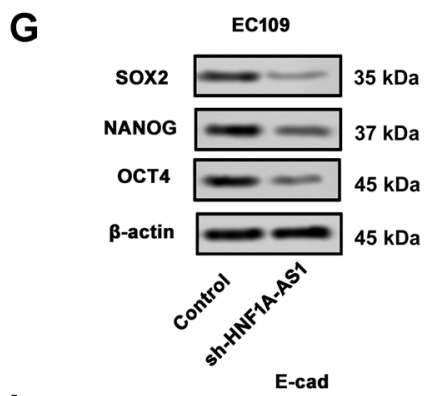
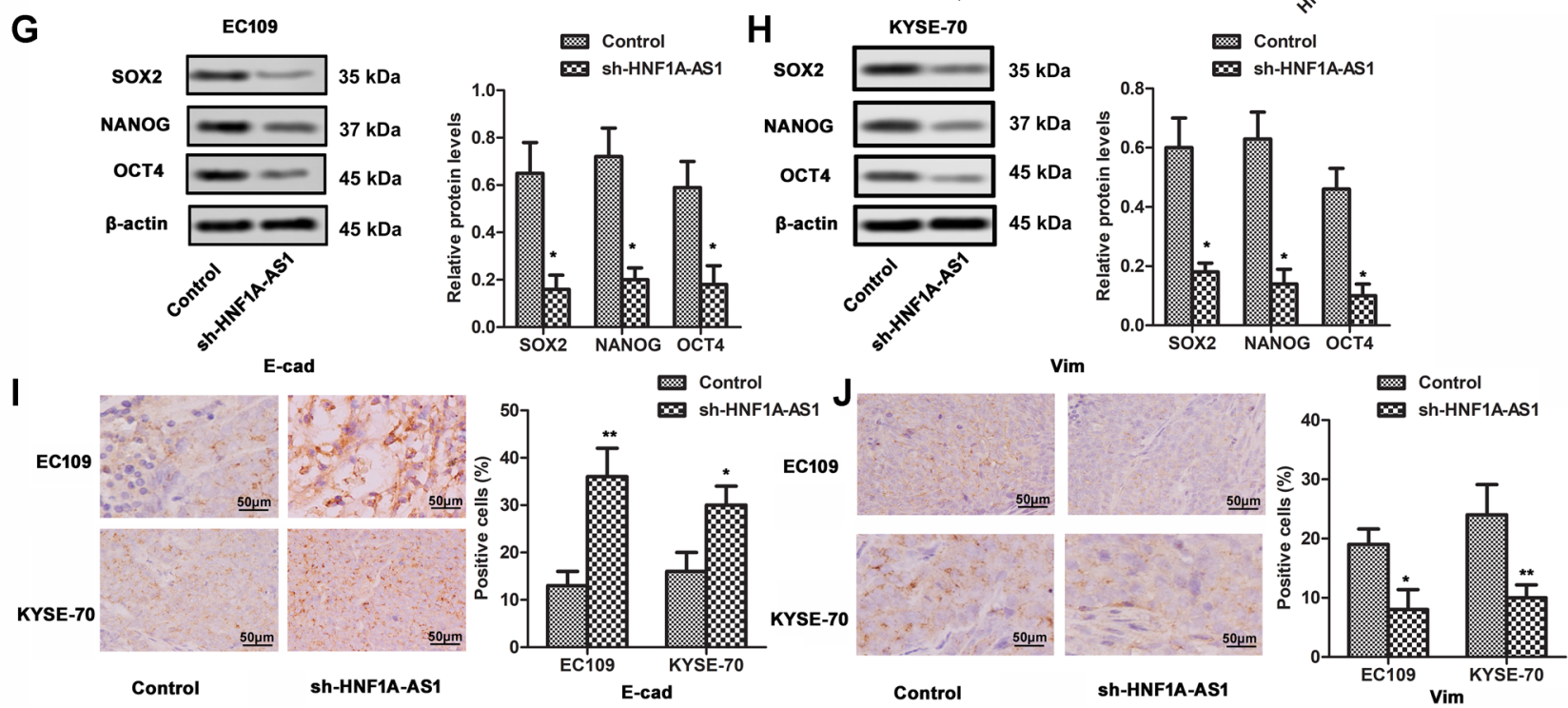

Figure 6. sh-HNF1A-AS1 inhibited tumor growth in vivo. A) Representative images of EC109 xenograft tissues are presented (n=5). B) Representative images of KYSE-70 xenograft tissues are presented $(n=5)$ C) Tumor weight of EC109 xenograft in each group is presented in the histogram. D) Tumor weight of KYSE-70 xenograft in each group is presented in the histogram E) The expression of HNF1A-AS1, TCF4, and miR-298 in EC109 tumor tissues was detected by qRT-PCR. F) The expression of HNF1A-AS1, TCF4, and miR-298 in KYSE-70 tumor tissues was detected by qRT-PCR. G) The expression of EMT-associated proteins in EC109 tumor tissues was evaluated by western blot analysis. H) The expression of EMT-associated proteins in KYSE-70 tumor tissues was evaluated by western blot analysis. I) The expression of stemness markers in EC109 tumor tissues was evaluated by immunohistochemistry. Representative images of each group are presented $(400 \times)$. J) The expression of stemness markers in KYSE-70 tumor tissues was evaluated by immunohistochemistry. Representative images of each group are presented $(400 \times) .{ }^{*} p<0.05$ vs. control group; ${ }^{* *} p<0.01$ vs. control group.

ability of the predicted miR-298 binding site on HNF1A-AS1 was confirmed by the luciferase activity assay; third, the RIP assays found that HNF1A-AS1 and miR-298 were in the same RISC; finally, the knockdown of HNF1A-AS1 (sh-HNF1AAS1) directly increased the expression of miR-298.

EMT is a highly conserved cellular process, in which loss of the epithelial marker E-cad and gain of the mesenchymal markers N-cad and Vim converts epithelial cells from noninvasive to invasive [18]. In Wu's study, they found HNF1AAS1 could induce the EMT process, thus promoting the proliferation and metastasis of lung adenocarcinoma [7]. And Mo et al. found that miR-298 could act as a target of lncR-LINC01287 to promote EMT in HCC cells [12]. In this study, some of EC109 and KYSE-70 cells transfected with
miR-298 inhibitor exhibited mesenchymal morphology, but sh-HNF1A-AS1 could reverse this morphologic change. Furthermore, our results also revealed that HNF1A-AS1 knockdown increased the expression of E-cad, while it decreased the expression of N-cad, Vim, and Snail. The miR-298 inhibitor had a reverse function on these proteins. These data suggested that HNF1A-AS1 and miR-298 both played important roles in EMT. HNF1A-AS1 might promote an EMT phenotype of EC109 cells by targeting miR-298.

The process of EMT is often associated with the acquirement of cancer stemness $[19,20]$. The term 'stemness' refers to the feature of cancer stem cells (CSCs) that control and maintain the stem cell state [21]. Increasing evidence suggested that CSCs with self-renewal properties might be 
a promising explanation for the recurrence and metastasis of cancer [22]. Thus, inhibiting the proliferation of CSCs has an important role in cancer treatment. ALDH activity is a specific marker of normal stem cells and various types of CSCs including ESCC $[23,24]$. Except for ALDH, SOX2, NANOG, and OCT4 are other makers of cancer stem cells [25]. In this study, we found that sh-HNF1A-AS1 could decrease the diameter of the sphere in the spheroid formation assays, reduce the number of $\mathrm{ALDH}^{+}$cells, and downregulate the expression of stemness markers in EC109 and KYSE-70 cells. Our results indicated that the knockdown of HNF1AAS1 could inhibit cancer stemness in ESCC cells.

TCF4 is one of the most important components in the Wnt/ $\beta$-catenin signaling pathway [26], which plays an important role in EMT and stemness [27]. Previous studies reported that some miRNAs, such as miR-155 [28], miR-495 [29], and miR-139-5p [30], could target TCF4 to regulate the Wnt/ $\beta$-catenin signaling pathway. In our study, the predictive bioinformatics analysis suggested that TCF4 was the most likely target of miR-298, which was confirmed by the luciferase reporter assays. And the expression of TCF4 was found decreased in ESCC cells co-transfected with sh-HNF1A-AS1 and increased in ESCC cells co-transfected with miR-298 inhibitor. The results above validated that TCF4 was a direct target of miR-298. HNF1A-AS1 may suppress miR-298 expression and consequently upregulates its target gene TCF4 in ESCC.

The in vivo effect of HNF1A-AS1 on ESCC was also explored in this study. Previously, it has been reported that a high level of HNF1A-AS1 in tumor tissues predicted poor prognosis [31, 32]. And knockdown of HNF1A-AS1 has been reported to impede tumor growth in tumor-bearing mice $[1,13,33]$. In support of previous concepts, sh-HNF1AAS1 was identified to suppress tumor growth in the ESCC xenograft mouse model in this study. And the inhibition role of sh-HNF1A-AS1 on EMT and stemness was also found in vivo. Our animal study data indicated that the knockdown of HNF1A-AS1 contributed to ESCC treatment.

In conclusion, HNF1A-AS1 was overexpressed in ESCC cell lines. miR-298 was validated as a direct target of HNF1A-AS1. Knockdown of HNF1A-AS1 could inhibit EMT and stemness by regulating the miR-298/TCF4 axis. These findings provide a novel HNF1A-AS1/miR-298/TCF4 pathway in ESCC and suggest HNF1A-AS1 could be a potential target for the occurrence, development, and treatment of human ESCC.

\section{References}

[1] WANG G, ZHAO W, GAO X, ZHANG D, LI Y et al. Hnf1a-as1 promotes growth and metastasis of esophageal squamous cell carcinoma by sponging mir-214 to upregulate the expression of sox-4. Int J Oncol 2017; 51: 657-667. https:// doi.org/10.3892/ijo.2017.4034
[2] HIRANO H, KATO K. Systemic treatment of advanced esophageal squamous cell carcinoma: Chemotherapy, molecular-targeting therapy and immunotherapy. Jpn J Clin Oncol 2019; 49: 412-420. https://doi.org/10.1093/jjco/hyz034

[3] GINN L, SHI L, MONTAGNA M, GAROFALO M. Lncrnas in non-small-cell lung cancer. Noncoding RNA 2020; 6: 25. https://doi.org/10.3390/ncrna6030025

[4] GUO X, ZHANG Y, LIU L, YANG W, ZHANG Q. Hnfla-as1 regulates cell migration, invasion and glycolysis via modulating mir-124/myo6 in colorectal cancer cells. Onco Targets Ther 2020; 13: 1507-1518. https://doi.org/10.2147/ott. s231249

[5] LIU HT, LIU S, LIU L, MA RR, GAO P. Egr1-mediated transcription of lncrna-hnf1a-as1 promotes cell-cycle progression in gastric cancer. Cancer Res 2018; 78: 5877-5890. https://doi.org/10.1158/0008-5472.can-18-1011

[6] ZHAO H, HOU W, TAO J, ZHAO Y, WAN G, et al. Upregulation of lncrna hnfla-as1 promotes cell proliferation and metastasis in osteosarcoma through activation of the wnt/ $\beta$-catenin signaling pathway. Am J Transl Res 2016; 8: 3503-3512.

[7] WU Y, LIU H, SHI X, YAO Y, YANG W et al. The long noncoding rna hnfla-as1 regulates proliferation and metastasis in lung adenocarcinoma. Oncotarget 2015; 6: 9160-9172. https://doi.org/10.18632/oncotarget.3247

[8] ZHANG G, AN X, ZHAO H, ZHANG Q, ZHAO H. Long non-coding rna hnfla-as1 promotes cell proliferation and invasion via regulating mir-17-5p in non-small cell lung cancer. Biomed Pharmacother 2018; 98: 594-599. https:// doi.org/10.1016/j.biopha.2017.12.080

[9] GUO N, SUN Q, FU D, ZHANG Y. Long non-coding rna ucal promoted the growth of adrenocortical cancer cells via modulating the mir-298-cdk6 axis. Gene 2019; 703: 26-34. https://doi.org/10.1016/j.gene.2019.03.066

[10] ARABSORKHI Z, GHARIB E, YAGHMOORIAN KHOJINI J, FARHADIEH ME, NAZEMALHOSSEINI-MOJARAD E et al. Mir-298 plays a pivotal role in colon cancer invasiveness by targeting pten. J Cell Physiol 2020; 235: 4335-4350. https://doi.org/10.1002/jcp.29310

[11] RUI X, WANG L, PAN H, GU T, SHAO S et al. LncRNA GAS6-AS2 promotes bladder cancer proliferation and metastasis via GAS6-AS2/miR-298/CDK9 axis. J Cell Mol Med 2019; 23: 865-876. https://doi.org/10.1111/jcmm.13986

[12] MO Y, HE L, LAI Z, WAN Z, CHEN Q et al. Linc01287/mir298/stat3 feedback loop regulates growth and the epithelialto-mesenchymal transition phenotype in hepatocellular carcinoma cells. J Exp Clin Cancer Res 2018; 37: 149. https:// doi.org/10.1186/s13046-018-0831-2

[13] MENG Q, WANG L, LV Y, WU J, SHI W. Deletion of hnflaas1 suppresses the malignant phenotypes of breast cancer cells in vitro and in vivo through targeting mirna-20a-5p/ trim32 axis. Cancer Biother Radiopharm 2020; 36: 23-35. https://doi.org/10.1089/cbr.2019.3168

[14] SALEM M, SHAN Y, BERNAUDO S, PENG C. miR-590$3 p$ Targets Cyclin G2 and FOXO3 to Promote Ovarian Cancer Cell Proliferation, Invasion, and Spheroid Formation. Int J Mol Sci 2019; 20: 1810. https://doi.org/10.3390/ ijms 20081810 
[15] SONG G, YANG Z, GUO J, ZHENG Y, SU X et al. Interactions among lncrnas/circrnas, mirnas, and mrnas in neuropathic pain. Neurotherapeutics 2020; 17: 917-913. https:// doi.org/10.1007/s13311-020-00881-y

[16] LIU HT, MA RR, LV BB, ZHANG H, SHI DB et al. Lncrnahnfla-as1 functions as a competing endogenous rna to activate pi3k/akt signalling pathway by sponging mir-30b-3p in gastric cancer. Br J Cancer 2020; 122: 1825-1836. https://doi. org/10.1038/s41416-020-0836-4

[17] FENG Z, WANG B. Long non-coding rna hnfla-as1 promotes cell viability and migration in human bladder cancer. Oncol Lett 2018; 15: 4535-4540. https://doi.org/10.3892/ ol.2018.7878

[18] PASTUSHENKO I, BLANPAIN C. Emt transition states during tumor progression and metastasis. Trends Cell Biol 2019; 29: 212-226. https://doi.org/10.1016/j.tcb.2018.12.001

[19] BASY S, CHERIYAMUNDATH S, BEN-ZE'EV A. Cell-cell adhesion: Linking wnt/ $\beta$-catenin signaling with partial emt and stemness traits in tumorigenesis. F1000Res 7 2018; 7 : 1488. https://doi.org/10.12688/f1000research.15782.1

[20] MITTAL V. Epithelial Mesenchymal Transition in Tumor Metastasis. Annu Rev Pathol 2018; 13: 395-412. https://doi. org/10.1146/annurev-pathol-020117-043854

[21] LIAO TT, YANG MH. Revisiting epithelial-mesenchymal transition in cancer metastasis: The connection between epithelial plasticity and stemness. Mol Oncol 2017; 11: 792-804. https://doi.org/10.1002/1878-0261.12096

[22] GUO F, YANG Z, KULBE H, ALBERS AE, SEHOULI J et al. Inhibitory effect on ovarian cancer aldh+ stem-like cells by disulfiram and copper treatment through aldh and ros modulation. Biomed Pharmacother 2019; 118: 109371. https:// doi.org/10.1016/j.biopha.2019.109371

[23] MORI Y, YAMAWAKI K, ISHIGURO T, YOSHIHARA K, UEDA $\mathrm{H}$ et al. Aldh-dependent glycolytic activation mediates stemness and paclitaxel resistance in patient-derived spheroid models of uterine endometrial cancer. Stem Cell Reports 2019; 13: 730-746. https://doi.org/10.1016/j.stemcr.2019.08.015

[24] JIA R, YANG L, YUAN X, KONG J, LIU Y et al. Gasc1 promotes stemness of esophageal squamous cell carcinoma via notch1 promoter demethylation. J Oncol 2019; 2019: 1621054. https://doi.org/10.1155/2019/1621054
[25] SHARIATI F, FAVAEDI R, RAMAZANALI F, GHORAEIAN $P$, AFSHARIAN $P$ et al. Increased expression of stemness genes rex-1, oct-4, nanog, and sox- 2 in women with ovarian endometriosis versus normal endometrium: A case-control study. Int J Reprod Biomed (Yazd) 2018; 16. https://doi. org/10.18502/ijrm.v16i12.3684

[26] ROBINSON KF, NARASIPURA SD, WALLACE J, RITZ EM, AL-HARTHI L. B-catenin and tcfs/lef signaling discordantly regulate il-6 expression in astrocytes. Cell Commun Signal 2020; 18: 93. https://doi.org/10.1186/s12964-020-00565-2

[27] YAN M, Li G, AN J. Discovery of small molecule inhibitors of the $\mathrm{wnt} / \beta$-catenin signaling pathway by targeting $\beta$-catenin/tcf4 interactions. Exp Biol Med (Maywood) 2017; 242: 1185-1197. https://doi.org/10.1177/1535370217708198

[28] ZHU F, LI H, LIU Y, TAN C, LIU X et al. Mir-155 antagomir protect against dss-induced colitis in mice through regulating th17/treg cell balance by jarid $2 /$ wnt $/ \beta$-catenin. Biomed Pharmacother 2020; 126: 109909. https://doi.org/10.1016/j. biopha.2020.109909

[29] ZHENG HE, WANG G, SONG J, LIU Y, LI YM et al. Microrna-495 inhibits the progression of non-small-cell lung cancer by targeting tcf 4 and inactivating wnt/ $\beta$-catenin pathway. Eur Rev Med Pharmacol Sci 2018; 22: 7750-7759. https:// doi.org/10.26355/eurrev_201811_16398

[30] DU F, CAO T, XIE H, LI T, SUN L et al. Kras mutation-responsive mir-139-5p inhibits colorectal cancer progression and is repressed by wnt signaling. Theranostics 2020; 10 : 7335-7350. https://doi.org/10.7150/thno.45971

[31] WANG YH, LIU YH, JI YJ, WEI Q, GAO TB. Upregulation of long non-coding rna hnfla-asl is associated with poor prognosis in urothelial carcinoma of the bladder. Eur Rev Med Pharmacol Sci 2018; 22: 2261-2265. https://doi. org/10.26355/eurrev_201804_14813

[32] ZHOU X, FAN YH, WANG Y, LIU Y. Prognostic and clinical significance of long non-coding rna hnfla-as1 in solid cancers: A systematic review and meta-analysis. Medicine (Baltimore) 2019; 98: e18264. https://doi.org/10.1097/ md.0000000000018264

[33] WU J, MENG X, JIA Y, CHAI J, WANG J et al. Long non-coding rna hnfla-as1 upregulates otx1 to enhance angiogenesis in colon cancer via the binding of transcription factor $\mathrm{pbx} 3$. Exp Cell Res 2020; 393: 112025. https://doi.org/10.1016/j. yexcr.2020.112025 